\title{
Circulating Serum Level of Visfatin in Patients with Endometrial Cancer
}

\author{
Aneta Cymbaluk-Płoska $\left(\mathbb{D},{ }^{1}\right.$ Anita Chudecka-Glaz $\mathbb{D},{ }^{1}$ Ewa Pius-Sadowska $\left(\mathbb{D},{ }^{2}\right.$ \\ Agnieszka Sompolska-Rzechuła, ${ }^{3}$ Bogusław Machaliński $\mathbb{D}^{2},{ }^{2}$ and Janusz Menkiszak ${ }^{1}$ \\ ${ }^{1}$ Department of Gynecological Surgery and Gynecological Oncology of Adults and Adolescents, \\ Pomeranian Medical University in Szczecin, Al. Powstańców Wielkopolskich 72, 70-111 Szczecin, Poland \\ ${ }^{2}$ General Pathology Department, Pomeranian Medical University in Szczecin, Al. Powstańców Wielkopolskich 72, \\ 70-111 Szczecin, Poland \\ ${ }^{3}$ Department of Mathematics Applications in Economy, West Pomeranian University of Technology, \\ Al. Piastów 17, 70-310 Szczecin, Poland \\ Correspondence should be addressed to Aneta Cymbaluk-Płoska; anetac@data.pl
}

Received 30 August 2017; Revised 22 October 2017; Accepted 28 November 2017; Published 4 January 2018

Academic Editor: Koichiro Wada

Copyright (c) 2018 Aneta Cymbaluk-Płoska et al. This is an open access article distributed under the Creative Commons Attribution License, which permits unrestricted use, distribution, and reproduction in any medium, provided the original work is properly cited.

\begin{abstract}
Obesity is a well-known factor that leads to many diseases including endometrial cancer. The adipose tissue is a heterogeneous organ of internal secretion. Visfatin is a newly discovered protein produced by fat tissues. The purpose of this work was to evaluate serum level concentrations of visfatin in patients with endometrial cancer based on clinical progression and histopathological tumor differentiation. The diagnostic capabilities of visfatin protein in high differentiation (FIGO III and IV) from a lower (FIGO I and II) clinical stage and prognostic degree of cell differentiation (G1 versus G2, G2 versus G3) on the basis of the analysis of the area under the ROC curve are as follows: $0.87,0.81$, and 0.86 . Significantly higher concentrations of visfatin have been observed in patients with invasion of the blood vessels $(p=0.02)$ and lymph node metastases $(p=0.01)$ in reference to the depth of infiltration of the endometrium $(p=0.004)$, as well as the size of the tumor $(p=0.003)$. Visfatin serum concentrations did not differ due to the invasion of the lymphatic vessels only. Visfatin seems to be a good marker of endometrial cancer progress. High visfatin serum level predicts poor prognosis in endometrial cancer patients.
\end{abstract}

\section{Introduction}

Lifestyle in developed countries has changed significantly in the last decades. The lack of physical activity and the easy access to high-calorie food contributed to the creation of an obese population [1]. Obesity is a well-known factor that conduces to many diseases including cancer $[2,3]$. The adipose tissue is an inhomogeneous organ of internal secretion. Many changes are to be observed in it, including aromatization of androgen to estrogen, which affects, due to their hyperfunction, a high frequency of such estrogenicrelated tumors as breast cancer and endometrial cancer. The adipose tissue discharges a variety of active proteins such as leptin, adiponectin, tumor necrosis factor, visfatin, resistin, omentin-1, and vaspin [4]. In 2009, Ye presented a study stating that patients characterized by long-lasting oxygen deficiency in the adipose tissue, as a consequence of the expansion of adipocytes, are affected by a long-standing inflammatory state of decreasing adiponectin, finally leading to the dysfunction of paracrine adipocytes [5]. In 2011, He et al. identified HIF-1 alpha and other mediators that cause hypoxia, conducing the release of free fatty acids from the adipose tissue. Free fatty acids directly affect the liver, and hence they are the causative factor of insulin immunity $[6,7]$. A recent study in 2015 suggested that hypoxia induces an increased discharge of VEGF and increases visfatin gene (3TC-L1) expression of adipocytes; all this affects oncogenesis. In light of the above studies, we were given an impulse to conduct the research using visfatin $[8,9]$. In our research, we would like to evaluate visfatin serum level in patients with different endometrial risk factors (obesity, diabetes mellitus type 2). However, the visfatin serum level in patients 
TABLE 1: Characteristics of patients with various risk factors of endometrial cancer.

\begin{tabular}{lc}
\hline Risk factor & $n$ \\
\hline PM/M & $29 / 99$ \\
\hline BMI $<25$ & 24 \\
BMI $25-30$ & 38 \\
BMI $>30$ & 66 \\
\hline WC $<100$ & 44 \\
WC $>100$ & 84 \\
\hline Glucose $<110$ & 40 \\
Glucose 110-126 & 36 \\
Glucose $>126$ & 52 \\
\hline DM-metformin & 24 \\
DM-insulin & 28 \\
\hline Mean systolic HA $<140 \mathrm{mmHg}$ & 49 \\
Mean systolic HA $>140 \mathrm{mmHg}$ & 79 \\
\hline
\end{tabular}

PM: premenopausal; M: postmenopausal; BMI: body mass index; WC: waist circumference; DM-metformin: diabetes mellitus treated with metformin; DM-insulin: diabetes mellitus treated with insulin; HA: arterial hypertension.

with endometrial cancer based on clinicopathological data is still unknown. In this study, we evaluated also serum concentrations of visfatin in patients with endometrial cancer based on clinical progression and histopathological tumor differentiation and whether serum visfatin levels can be associated with poorer patient prognosis.

\section{Materials and Methods}

2.1. Clinicopathological Data. The study included 128 patients who were admitted due to vaginal bleeding. All patients signed informed consent to participate in the study. The study protocol was approved by the Ethical Committee of Pomeranian Medical University.

Detailed division is presented in Tables 1 and 2.

Following surgical treatment and with the results of histopathological examination, patients were assorted into two groups:

(1) Patients with endometrial cancer: $n=78$.

(2) Patients with normal endometrium: $n=63$.

Among the group of patients with endometrial cancer, we identified the following:

(1) 64 patients with endometrial cancer.

(2) 14 patients with nonendometrial cancer.

Patients from the endometrial cancer group were divided according to tumor grading into subgroups $\mathrm{G} 1=17, \mathrm{G} 2=41$, and G3 $=20$, as well as depending on clinical tumor staging:

(1a) FIGO I and II patients, $n=62$.

(2a) FIGO III and IV patients, $n=16$.

The study assessed the depth of myometrium infiltration, size of the tumor, invasion of blood vessels and lymphatic vessels, and metastasis to lymph nodes. Table 3 provides the exact distribution of the individual subgroups.
TABLE 2: Concentration of visfatin in serum depending on the patient's histopathological diagnosis.

\begin{tabular}{lcc}
\hline & Endometrial cancer & Normal endometrium \\
\hline Age & 54.6 & 52.9 \\
Average height & $166.9 \pm 18.9$ & $172 \pm 17.2$ \\
{$[\mathrm{~cm}]$} & $94.7 \pm 16.5$ & $89 \pm 15.7$ \\
Average weight & $31.3 \pm 5.2$ & $29.8 \pm 4.7$ \\
{$[\mathrm{~kg}]$} & $106.5 \pm 10.8$ & $103.2 \pm 8.99$ \\
BMI & $146 / 93$ & $138 / 84$ \\
WC & $77.2 \%$ & $55.2 \%$ \\
HA &
\end{tabular}

BMI: body mass index; WC: waist circumference; HA: arterial hypertension; DM type 2: diabetes mellitus type 2 .

2.2. Laboratory Measurements. Five milliliters of blood was collected from each patient (for the determination of visfatin levels on the occasion of routine preoperative testing) and centrifuged. The serum was subsequently frozen and stored at $-70^{\circ} \mathrm{C}$.

2.3. Multiplex Immunoassay. Visfatin concentrations were quantified in serum/plasma by multiplex fluorescent beadbased immunoassays (Luminex Corporation, Austin, TX, USA) using commercial Human Bio-Plex Pro Diabetes Assays (Bio-Rad, Hercules, CA, USA). $50 \mu \mathrm{L}$ of antibody capture bead solution was added to each well of the assay plate and the plate was washed two times with $100 \mu \mathrm{L}$ of a wash buffer. After washing, $50 \mu \mathrm{L}$ of each blank, standard, and sample were added to the plate, and the plate was incubated with agitation for 1 hour at room temperature and in the dark. After this step, the well was washed with $100 \mu \mathrm{L}$ of a wash buffer three times using a handheld magnet. $25 \mu \mathrm{L}$ of a detection antibody cocktail was pipetted to each well and the plate was sealed and incubated at room temperature for 30 minutes on a plate shaker. After washing, $50 \mu \mathrm{L}$ of a streptavidin-phycoerythrin mixture was added to the plate and incubated with agitation for 10 minutes in the dark. Finally, after washing, the microspheres in each well were resuspended in $125 \mu \mathrm{L}$ assay buffer and shaken at room temperature for 30 seconds. The plate was then read and analyzed on the Luminex analyzer and analyte concentrations were determined from five different standard curves showing MFI (median fluorescence intensity) versus protein concentration.

2.4. Statistical Analysis. Statistical analysis was performed using STATISTICA 10.0 PL program. The descriptive characteristic of the examined population of patients was prepared, determining minimum, maximum, mean, and median values. Because the distributions of the analyzed features to compare mean values are not normal distributions, median positional parameters were used as well as nonparametric tests (Kruskal-Wallis test and post hoc Dunn test for comparison of three groups and Mann-Whitney $U$ test for comparison of two groups). For the selected groups, the receiver operating characteristic (ROC) curves were obtained and the area under the curve (AUC) was calculated with 
TABLE 3: Visfatin concentration in serum depending on risk factors for endometrial cancer.

\begin{tabular}{|c|c|c|c|c|c|c|}
\hline & Mean & Median & Min. & Max. & SD & $p$ \\
\hline $\mathrm{PM} / \mathrm{M}$ & $14.9 / 17.1$ & $15.3 / 18.4$ & $7.1 / 8.4$ & $20.9 / 23.3$ & $1.08 / 1.28$ & NS \\
\hline $\begin{array}{l}\text { BMI }<25 \\
\text { BMI } 25-30\end{array}$ & $9.2 / 14.3$ & $10.1 / 16$ & $6.8 / 5.9$ & $18.2 / 31.2$ & $2.01 / 3.3$ & 0.02 \\
\hline $\begin{array}{l}\text { BMI 25-30 } \\
\mathrm{BMI}>30\end{array}$ & $14.3 / 21.1$ & $16 / 23.4$ & $5.9 / 7.8$ & $31.2 / 36.7$ & $3.3 / 3.6$ & 0.001 \\
\hline $\mathrm{WC}<100 / \mathrm{WC}>100$ & $12.2 / 19.4$ & $14.2 / 20.6$ & 7.1/11.1 & $19.5 / 34.2$ & $1.8 / 2.7$ & 0.003 \\
\hline Glucose $<110 /$ glucose 110-126 & $13.6 / 19.2$ & $15.1 / 21.8$ & $11.4 / 13.9$ & $20.02 / 27.9$ & $1.03 / 1.6$ & 0.04 \\
\hline Glucose $110-126 /$ glucose $>126$ & $19.2 / 28.7$ & $21.8 / 31.2$ & $13.9 / 17.8$ & $27.9 / 36.1$ & $1.6 / 2.4$ & 0.01 \\
\hline DM-metformin/DM-insulin & $21.3 / 28.4$ & $22.3 / 30.4$ & $16.2 / 18.7$ & $25.6 / 35.3$ & $2.6 / 3.4$ & 0.03 \\
\hline Mean systolic $\mathrm{HA}<140 \mathrm{mmHg} /$ mean systolic $\mathrm{HA}>140 \mathrm{mmHg}$ & $18.2 / 23.4$ & $19.7 / 24.5$ & $15.1 / 16.2$ & $22.2 / 28.4$ & $1.9 / 2.3$ & NS \\
\hline
\end{tabular}

PM: premenopausal; M: postmenopausal; BMI: body mass index; WC: waist circumference; DM-metformin: diabetes mellitus treated with metformin; DMinsulin: diabetes mellitus treated with insulin; HA: arterial hypertension.

95\% confidence intervals according to the nonparametric method of DeLong. A $p$ value of $<0.05$ was considered as statistically significant. The study variables were analyzed using the logistic regression model.

Logistic regression coefficients may be determined using the maximum likelihood method or the generalized least squares method. Due to the nonlinearity of the model in relation to the independent variables and parameters, the logistic model is transformed into a linear regression model using logarithmic transformation. To this end, the concept of odds ratio (OR) is introduced as the ratio between the likelihood of a particular event and the likelihood of that event not happening. Therefore, the odds ratio is used to express the factor of the increase or the decrease in the likelihood of a particular event upon a unit change in the independent variable (with fixed values of the remaining independent variables). Kaplan-Meier survival analysis and log-rank test were used to assess the impact of visfatin on overall survival and progression time. The following cut-off values were determined for baseline visfatin levels: median and level of $20.7 \mathrm{ng} / \mathrm{ml}$. Single and multifactorial analysis was performed using the Cox regression model. Multifactorial Cox analysis parameters included age, FIGO, grading, median, and cut-off $20.7 \mathrm{ng} / \mathrm{ml}$ visfatin levels.

\section{Results}

3.1. Comparative Analysis of the Treatment Groups. The mean concentrations of individual proteins in serum are presented in Table 3. There is evidence of statistically significant lower mean concentrations of visfatin in patients with correct level of glycemia, without diabetes mellitus type 2 in relation to mean concentrations in patients with obesity or overweight, with an elevated level of glycemia, as well as diabetes mellitus type 2. Statistically significant correlations were found between WC patients and visfatin concentration, $r=0.882$ for $p=0.002$. The study has shown statistically significant differences in mean visfatin concentrations between patients with diabetes mellitus type 2 treated with metformin and patients with diabetes mellitus type 2 treated with insulin, respectively $(21.3 \mathrm{ng} / \mathrm{ml}, 28.4 \mathrm{ng} / \mathrm{ml})$. In contrast, the correlation between blood pressure and the mean values of visfatin protein was not found. The results are summarized in detail in Table 3.

We have found statistically significant differences in mean serum visfatin concentrations between a group of patients with endometrial cancer and patients with bleeding disorder and unchanged endometrium. The date was found among all analyzed women $(p=0.002)$ as well as after having taken into account the distribution depending on the hormonal status (premenopausal, $p=0.003$; postmenopausal, $p=0.0002$ ). The characteristics of the patients and the mean values of the test protein along with the status of hormonal distribution of patients are presented in Table 3.

3.2. Comparative Analysis due to the Risk Factors. Table 4 shows serum concentrations of visfatin due to prognostic factors together with comparisons. Having analyzed all currently recognized adverse prognostic risk factors of endometrial cancer, it has been stated that between two types of cancer (endometrioid endometrial cancer versus nonendometrioid endometrial cancer) there are no statistically significant differences. Visfatin serum concentrations did not differ due to the invasion of lymphatic vessels. However, statistically significant differences between the well-differentiated cancer group and a mean differentiated cancer group at mean concentration $(17.3 \mathrm{ng} / \mathrm{ml}, 22.2 \mathrm{ng} / \mathrm{ml})$ and between $\mathrm{G} 2$ and G3 $(22.2 \mathrm{ng} / \mathrm{ml}, 31.8 \mathrm{ng} / \mathrm{ml})$ have been proved (Figure 1). Significantly higher concentrations of visfatin have been observed in patients with highly advanced cancers versus lower advanced cancers, $p=0.0002$, and in the case of the invasion of blood vessels, $p=0.02$, and lymph node metastases, $p=0.01$, in reference to the depth of infiltration of the endometrium, $p=0.004$, as well as the size of the tumor, $p=0.003$.

3.3. The ROC Analysis and Sensitivity and Specificity of the Test. In order to evaluate the diagnostic value of visfatin, the ROC curves were established and the area under the ROC curve (AUC) was assessed. For visfatin, the AUC values were 0.89 for all the analyzed patients, 0.87 for premenopausal women, and 0.92 for postmenopausal women. The diagnostic capabilities of visfatin protein in high differentiation (FIGO 
TABLE 4: Visfatin concentration in serum, depending on staging and grading group of endometrial cancer.

\begin{tabular}{|c|c|c|c|c|c|c|}
\hline & Mean & Median & Min. & Max. & $\mathrm{SD}$ & $p$ \\
\hline Endometrioid/nonendometrioid & $19.8 / 23.1$ & $20.4 / 24.3$ & $13.4 / 16.1$ & $22.2 / 29.6$ & $2.4 / 2.9$ & NS \\
\hline G1/G2 & $17.3 / 22.2$ & $18.3 / 23.9$ & $13.2 / 10.2$ & $19.9 / 31.3$ & $1,8 / 3.7$ & 0.03 \\
\hline G2/G3 & $22.2 / 31.8$ & $23.9 / 33.8$ & $10.2 / 14.6$ & $31.3 / 41.2$ & $3.7 / 4.2$ & 0.002 \\
\hline FIGO I and II/FIGO III and IV & $16.8 / 32.4$ & $17.1 / 33.5$ & $11.8 / 15.4$ & $22.1 / 40.9$ & $2.03 / 4.2$ & 0.0002 \\
\hline Tumor size $<2 \mathrm{~cm}$ or $>2 \mathrm{~cm}$ & $19.1 / 33.3$ & $20.02 / 35.1$ & $15.2 / 17.1$ & $25.2 / 42.1$ & $2.4 / 4.3$ & 0.003 \\
\hline Infiltration of the myometrium: superficial/deep & $18.4 / 30.6$ & 19.1/31.8 & $11.2 / 17.3$ & $25.1 / 38.7$ & $2.2 / 3.6$ & 0.004 \\
\hline Lymph vessels invasion $+/-$ & $25.5 / 22.3$ & $27 / 23.1$ & $17.2 / 16.5$ & $29.1 / 26.6$ & $2 / 2.2$ & NS \\
\hline Vascular invasion $+/-$ & $29.8 / 21.4$ & $30.3 / 22.3$ & $16.7 / 19.1$ & $39.2 / 30$ & $4.1 / 2.8$ & 0.02 \\
\hline Lymph nodes metastasis +/- & $39.1 / 21.2$ & $40.01 / 22.6$ & $16.2 / 18.4$ & $46.1 / 29.2$ & $4.7 / 2.1$ & 0.01 \\
\hline
\end{tabular}

TABLE 5: Multivariate logistic regression analysis.

\begin{tabular}{lccc}
\hline & OR & $95 \%$ CI & $p$ \\
\hline BMI $\left[\mathrm{kg} / \mathrm{m}^{2}\right]$ & 1.2 & $1.06-1.38$ & 0.01 \\
WC & 1.03 & $0.98-1.18$ & 0.02 \\
Glucose level & 0.79 & $0.66-0.97$ & 0.32 \\
DM type 2 & 0.87 & $0.73-1.03$ & 0.56 \\
Visfatin $[\mathrm{ng} / \mathrm{ml}]$ & 1.23 & $1.14-1.49$ & 0.01 \\
Age & 1.06 & $0.97-1.18$ & 0.04 \\
HA & 0.43 & $0.28-0.69$ & 0.59 \\
\hline
\end{tabular}

BMI: body mass index; WC: waist circumference; DM type 2: diabetes mellitus type 2; HA: arterial hypertension.

III and IV) from a lower (FIGO I and II) clinical stage and prognostic degree of cell differentiation (V1 versus G2, G2 versus G3) on the basis of the analysis of the area under the ROC curve are as follows: $0.87,0.81$, and 0.86 . This data has been presented in Figures 2-5. Having taken into account the hormonal status, the values of sensitivity and specificity for visfatin are presented in Table 6 . We found greater sensitivity than specificity for visfatin in all analyzed women (88\% versus $79 \%$ ): for postmenopausal women, $90 \%$ versus $83 \%$, in comparison with the sensitivity and specificity for premenopausal women, $85 \%$ versus $73 \%$.

3.4. Multivariate Logistic Regression Analysis. Having applied multivariate logistic regression analysis for the risk of the development of endometrial cancer, in the final model, independent risk factors were found: glucose level, BMI, $\mathrm{WC}$, and visfatin level. Classification determined on the basis of the cut-off values for visfatin $(20.7 \mathrm{ng} / \mathrm{ml})$ gives $94.38 \%$ correctly classified cases, out of which correctly classified "Yes" is $72.94 \%$ (sensitivity [95\% CI] $=72.94 \%$ [ $48.81 \%$; $91.02 \%]$ ) and "No" is $97.74 \%$ (specificity $[95 \% \mathrm{CI}]=97.74 \%$ [85.46\%; 99.48\%]) (Table 5).

3.5. Patients' Survival Time Evaluation Using the Kaplan-Meier Curves and Cox Proportional Hazards Regression. Statistical analysis performed using the Kaplan-Meier survival curves and log-rank test revealed that there are statistically significant correlations between the median and $20.7 \mathrm{ng} / \mathrm{ml}$ cut-off visfatin levels with recurrence-free survival of patients ( $p=$ $0.00002 / p=0.02552$ ) (Figure 6). High baseline visfatin levels
TABLE 6: Sensitivity and specificity for visfatin.

\begin{tabular}{lcc}
\hline & Sensitivity & Specificity \\
\hline Visfatin & $88 \%$ & $79 \%$ \\
Visfatin PM & $85 \%$ & $73 \%$ \\
Visfatin M & $90 \%$ & $83 \%$ \\
\hline
\end{tabular}

PM: premenopausal; M: postmenopausal.

were shown to be correlated with shorter overall survival times in endometrial cancer patients $(p=0.0001)$ (Figure 7). Cox proportional hazards regression analysis with a single group variable revealed a correlation between the recurrencefree survival and clinical tumor staging $(p=0.0003)$. Univariate Cox regression analyses revealed correlations between overall survival and age $(p=0.038)$, tumor staging $(p=$ $0.027)$, and above-median visfatin levels $(p=0.0056)$. In the RFS model, multivariate Cox regression analysis with age, grading, clinical stage, and above-median visfatin levels as the group variables revealed that patients with above-median visfatin levels had shorter RFS times compared to patients with below-median visfatin levels $(p=0.023, \mathrm{HR}=0.99)$. In the univariate OS model, both median visfatin level and cut-off baseline visfatin level $(20.7 \mathrm{ng} / \mathrm{ml})$ were significant variables. A correlation was demonstrated between median visfatin levels and overall survival of patients $(p=0.03$, HR $=0.97$. The higher the visfatin level above $20.7 \mathrm{ng} / \mathrm{ml}$, the shorter the overall survival of patients.

\section{Discussion}

Obesity is a major health problem in the population in most highly developed countries. Approximately $40 \%$ of postmenopausal women have a problem with overweight or obesity. There is a known fact that certain cancers correlate with obesity; these include, for example, endometrial cancer $[10,11]$. Diabetes mellitus type 2 and insulin resistance associated with it are well-known risk factors for endometrial cancer. New active biological factors that are secreted in the visceral adipose tissue and aid the formation of tumors are investigated. This factor may be visfatin. Our research has confirmed the positive correlation between visfatin and obesity/diabetes mellitus type 2 and evidence of neoplastic changes in every analyzed patient group. Visfatin as a 

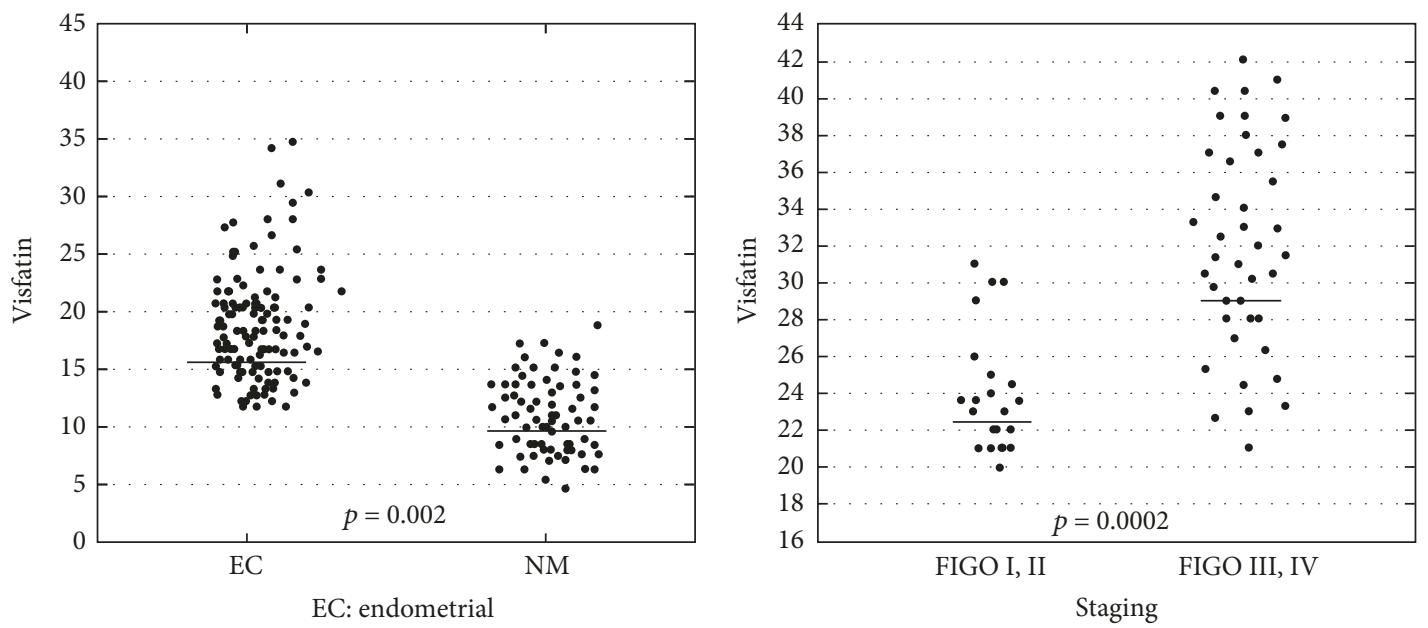

NM: normal endometrium
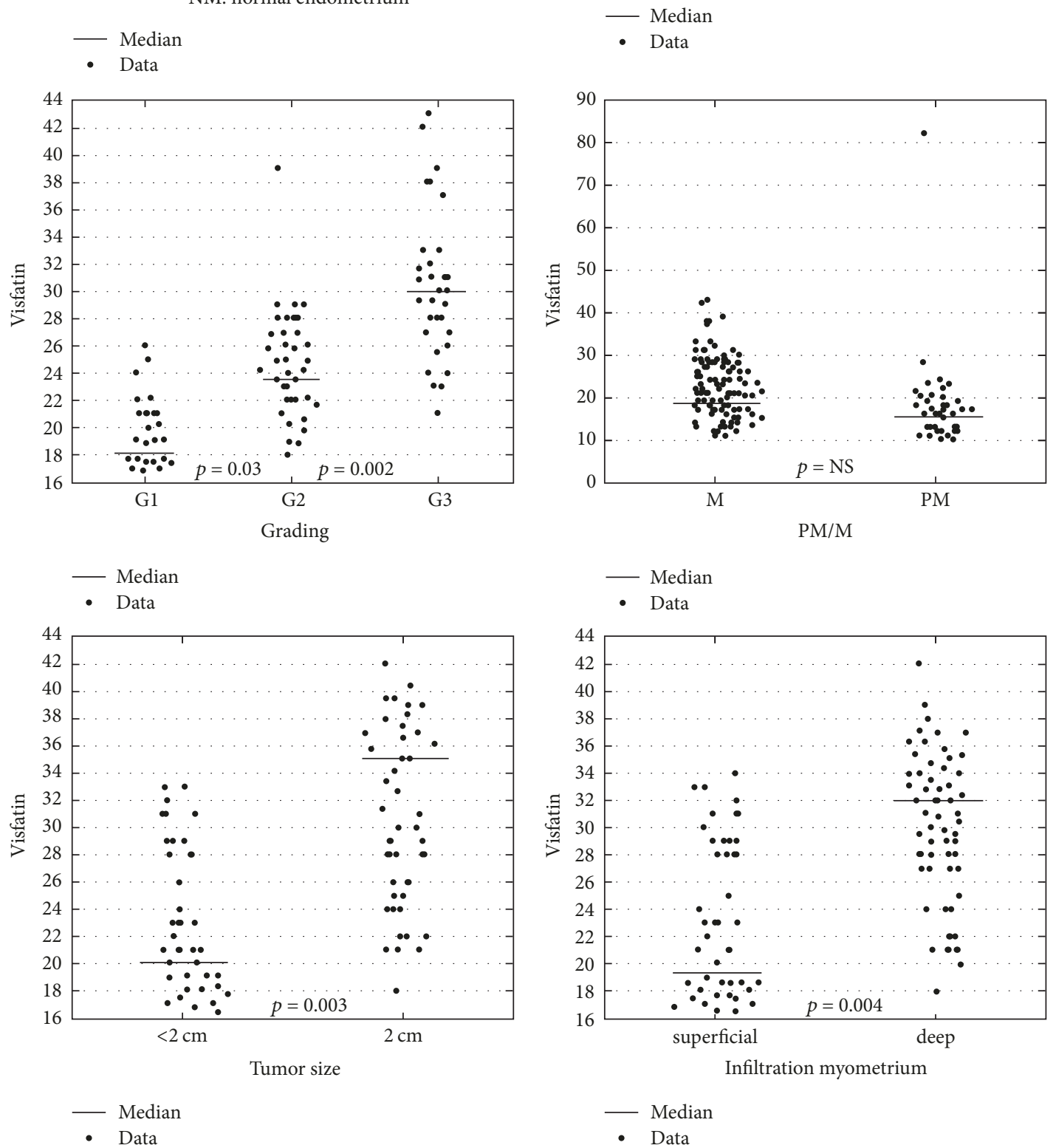

Median
- Data

- Data

FIGURE 1: Visfatin serum levels comparison in individual groups. 


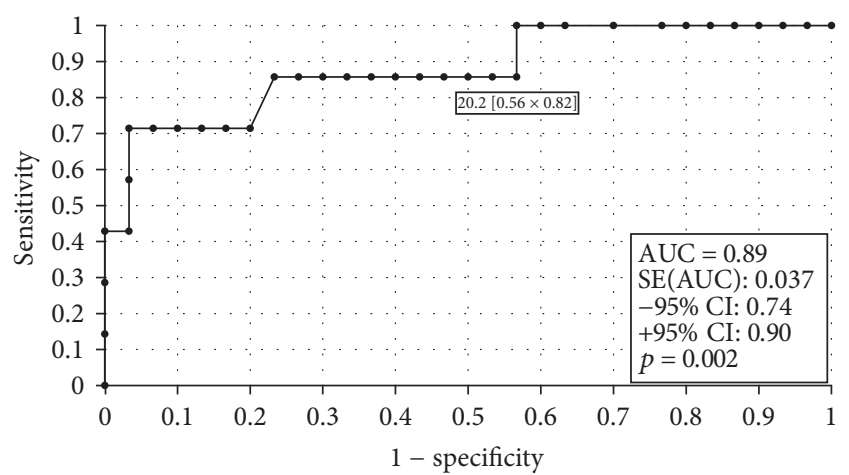

FIgURE 2: The ROC curves for visfatin proteins in women. The analysis compared endometrial cancer patients to patients with benign endometrial lesions.

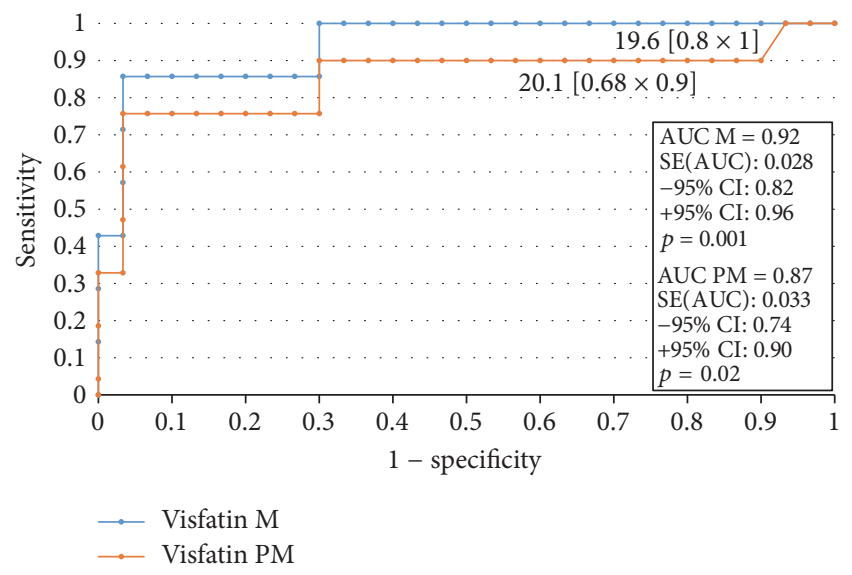

FIGURE 3: The ROC curves for visfatin proteins in women. The analysis compared endometrial cancer patients to patients with benign endometrial lesions depending on the hormonal status.

cytokine was identified for the first time by Samal et al. in 1994 [12].

Sheu et al. emphasized that a significant weight reduction correlates with a decrease of serum levels of leptin and visfatin in patients by 17.2 and $50.2 \%$, respectively, and with an increased level of adiponectin by $32.2 \%$ [13]. A metaanalysis conducted by Chang et al. proves that the increase of visfatin level is evident in obesity, diabetes mellitus type 2, and metabolic syndrome [14]. The characteristic common for the diseases mentioned above is the phenomenon of insulin resistance, whereby in a sequence of events, insulin resistance, hyperinsulinemia, the mechanism of downregulation, and the number of receptors for insulin decreased. It has also been shown that visfatin is an adipokine with proinflammatory and immunomodulatory properties.

It has the ability to activate human leukocytes, induces the synthesis of proinflammatory cytokines and adhesion molecules, regulates the maturation of leukocytes $B$, and inhibits apoptosis of neutrophils [15-17]. It also has a hepatoprotective effect in nonalcoholic fatty liver disease (NAFLD) [18]. It assumes a role in the regulation of obesity-related inflammation [19].

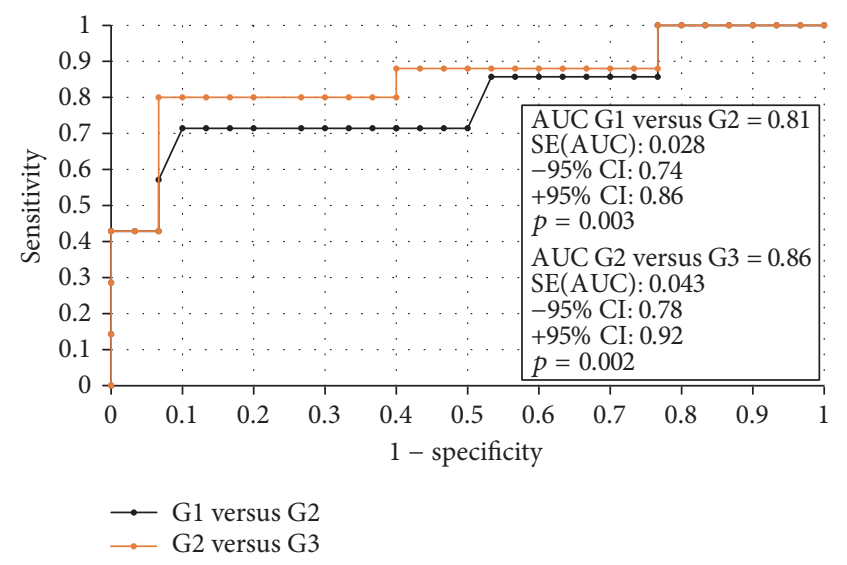

FIGURE 4: The ROC curves for visfatin proteins depending on endometrial cancer grading.

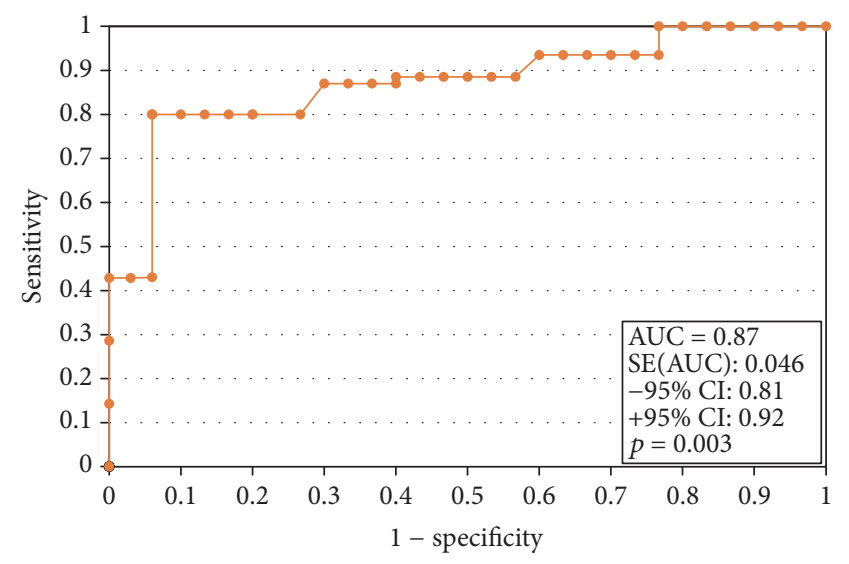

FIGURE 5: The ROC curves for visfatin proteins depending on endometrial cancer staging.

It is produced by a number of cells and its elevated concentration can be found in many acute and chronic inflammatory diseases [20]. The serum visfatin concentration is correlated with the metabolic syndrome [21].

Studies have shown that visfatin/PBEF is identical to nicotinamide (Nampt) phosphoribosyltransferase, which regulates the intracellular synthesis of adenine dinucleotide (NAD) from nicotinamide and thus energy processes in the cell [16]. In addition, visfatin increases the synthesis of nicotinamide mononucleotide (NMN), which stimulates the $\beta$ pancreatic cells for insulin production.

As we know, visfatin stimulates the synthesis of TNF and IL-6, which could suggest its adverse effect on the development of insulin resistance [15].

In addition, by indirectly stimulating the synthesis of NF$\kappa \mathrm{b}$ and increasing the production of free radicals, visfatin may additionally contribute to the rise of insulin resistance in obese patients $[15,22]$. Nampt/visfatin acts both intracellularly and extracellularly, regulating many biological functions in the body, including the metabolism of NAD, carcinogenesis, inflammation, and stress. 

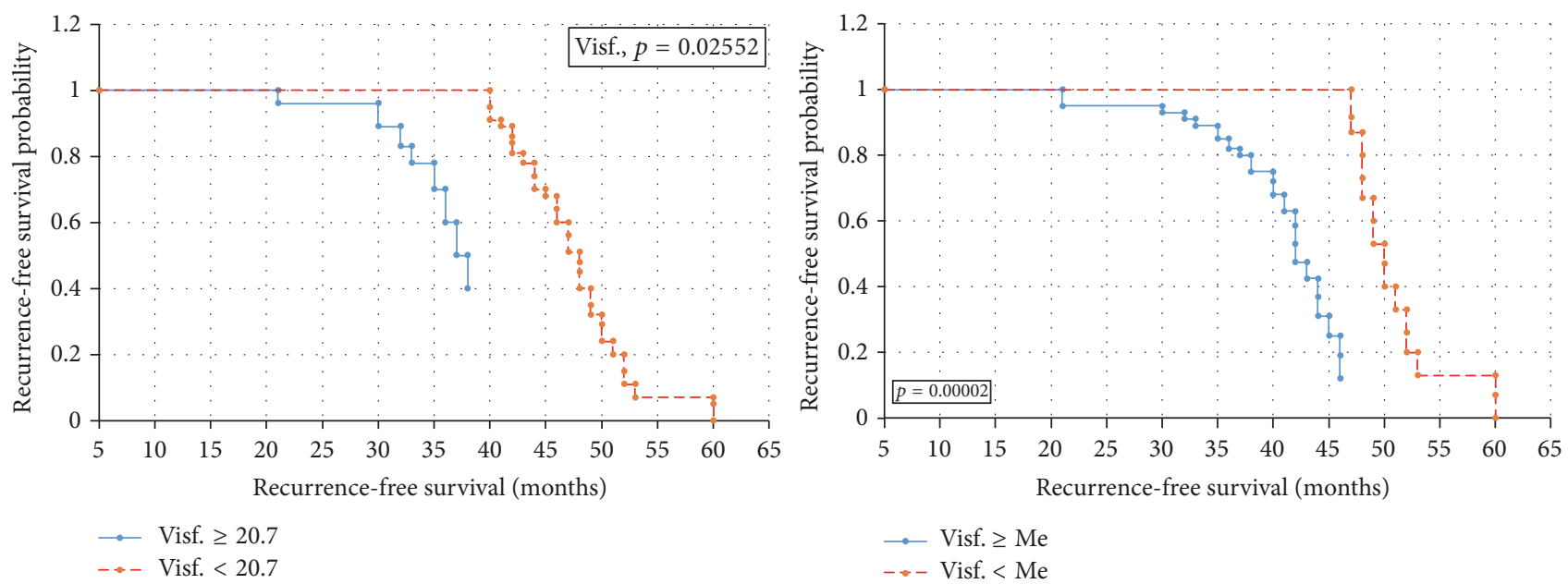

FIGURE 6: Kaplan-Meier recurrence-free survival curves for endometrial cancer patients based on serum visfatin level.

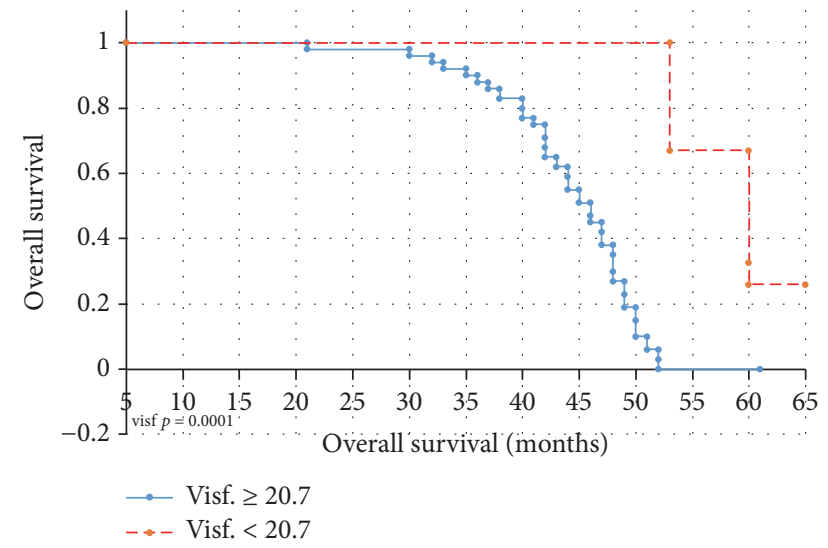

FIGURE 7: Kaplan-Meier overall survival curves for endometrial cancer patients based on serum visfatin level.

Despite showing the multifunctionality of proteins, there are few reports emphasizing its role in carcinogenesis. Previous reports indicate that visfatin can function as a growth factor or as a cytokine in molecular mechanisms, including signaling pathways PI3K/Act, EQ1/2, and STAT3 [23, 24]. Exogenous administration of recombinant visfatin increases the proliferation of cells in human cells of breast cancer MCF-7 [22]. In a research, Lee et al. found that the high expression of visfatin in breast cancer tissue was associated with higher progression of breast cancer and poor prognosis [25]. Folgueira et al. also concluded that hyperexpression of visfatin may predict a poor response to doxorubicin-based chemotherapy for breast cancer [26].

The research by Wang et al. has revealed that there is an increased visfatin expression in endometrial cancer cell lines, promoting proliferation by activating the signaling pathways PI3K/Akt and MAPK/ERK1/2 [27]. Our research has proved a statistically significant increase of visfatin levels in patients with endometrial cancer compared to patients with normal endometrium. Similar results are presented by Ilhan et al. They showed the mean concentration of the EC group for visfatin at $14.9 \mathrm{ng} / \mathrm{ml}$ and in the control group at $8.1 \mathrm{ng} / \mathrm{m}$ [28]. In our study, the mean concentrations in both groups were slightly higher and were as follows: $15.9 \mathrm{ng} / \mathrm{ml}$ for the EC and $9.5 \mathrm{ng} / \mathrm{ml}$ for the control group. Evidence of the higher concentrations of visfatin protein in endometrial cancers is also presented in the studies of Avcioglu et al. [29-31]. Only Luhn et al., who evaluated the serum level concentrations of all adipokines in patients with endometrial cancer, proved the higher visfatin levels, but without statistical significance [32]. The authors themselves stressed that the results require confirmation due to the small number of patients tested. Tian et al. demonstrated that the level of visfatin expression in tissues corresponds to the serum concentration of visfatin in patients with endometrial cancer [31]. The role of visfatin in predicting clinical tumor stage was analyzed by Ilhan et al., by specifying it as an invasion of the myometrium [28]. Serum concentration of visfatin was far higher in patients with infiltration exceeding $1 / 2$ of the myometrium. The cutoff point for visfatin for the clinical stage of IB tumor was $26.8 \mathrm{ng} / \mathrm{ml}$. Ilhan et al. also investigated visfatin concentration depending on changes in the lymph nodes [28]. They found no statistically significant differences. In our study, the serum concentration of visfatin was higher but statistically insignificant. Serum concentration of visfatin in our study was evaluated also in occupied lymphatic and blood vessels as well as depending on the histopathological differentiation of the tumor. The higher mean serum concentration of visfatin has been found in the case of larger tumor size as lower histological differentiation of tumor and occupied lymphatic vessels.

Similar results are presented by Tian et al. who suggested that high levels of visfatin were associated with higher clinical staging and depth of myometrium infection. The same authors emphasized, however, that the expression of visfatin in the endometrium cancer tissue was not correlated with the degree of histopathological differentiation and lymph nodes metastasis. It may be a prognostic factor in patients with endometrial cancer. In our study, we found that high visfatin serum level will correlate with faster resumption and 
shorter survival time of patients with endometrial cancer. The same results were presented by Tian et al. who, analyzing the relationship between visfatin expression and total patient survival time, stated that high visfatin expression is believed to be associated with poor prognosis of patients, shorter survival time, and shorter time of recurrence.

Avcioglu et al. have demonstrated that the visfatin serum level can be treated as a risk factor for endometrial cancer on a par with patient age, BMI, and diabetes mellitus type 2 [29]. Using multivariate logistic regression analysis in our research for the development of endometrial cancer risk, in the final model, independent risk factors like glucose level, BMI, WC, and the visfatin level were found. The odds ratio of endometrial cancer when increasing the visfatin concentration of $1 \mathrm{ng} / \mathrm{ml}$ is 1.23 .

\section{Conclusion}

(1) Higher visfatin levels were found in obesity and type 2 diabetes. Both diseases are conducive to endometrial cancer.

(2) Visfatin seems to be a good marker of endometrial cancer progression.

(3) High serum visfatin levels can be associated with poorer patient prognosis.

\section{Abbreviations}

$\begin{array}{ll}\text { OR: } & \text { Odds ratio } \\ \text { 95\% CI: } & \text { 95\% confidence intervals } \\ \text { PM: } & \text { Premenopausal } \\ \text { M: } & \text { Postmenopausal } \\ \text { SD: } & \text { Standard deviation } \\ \text { WC: } & \text { Waist circumference } \\ \text { HA: } & \text { Arterial hypertension } \\ \text { BMI: } & \text { Body mass index } \\ \text { ROC: } & \text { Receiver operating characteristic } \\ \text { AUC: } & \text { Area under the curve } \\ \text { FIGO: } & \text { Fédération Internationale de Gynécologie } \\ & \text { et d'Obstétrique (International Federation } \\ & \text { of Gynecology and Obstetrics) } \\ \text { G [1-3]: } & \text { Grading } \\ \text { DM type 2: } & \text { Diabetes mellitus type 2 } \\ \text { DM-metformin: } & \text { Diabetes mellitus treated with metformin } \\ \text { DM-insulin: } & \text { Diabetes mellitus treated with insulin } \\ \text { OS: } & \text { Overall survival } \\ \text { RFS: } & \text { Recurrence-free survival. }\end{array}$

\section{Conflicts of Interest}

The authors declare that they have no conflicts of interest regarding the publication of this paper.

\section{References}

[1] D. Aune, A. Sen, and L. J. Vatten, "Hypertension and the risk of endometrial cancer: A systematic review and meta-analysis of case-control and cohort studies," Scientific Reports, vol. 7, Article ID 44808, 2017.

[2] J. Ni, T. Zhu, L. Zhao et al., "Metabolic syndrome is an independent prognostic factor for endometrial adenocarcinoma," Clinical and Translational Oncology, vol. 17, no. 10, pp. 835-839, 2015.

[3] A. G. Renehan, M. Tyson, M. Egger, R. F. Heller, and M. Zwahlen, "Body-mass index and incidence of cancer: a systematic review and meta-analysis of prospective observational studies," The Lancet, vol. 371, no. 9612, pp. 569-578, 2008.

[4] A. Booth, A. Magnuson, J. Fouts, and M. Foster, "Adipose tissue, obesity and adipokines: role in cancer promotion," Hormone Molecular Biology and Clinical Investigation, vol. 21, no. 1, pp. 57-74, 2015.

[5] J. Ye, "Emerging role of adipose tissue hypoxia in obesity and insulin resistance," International Journal of Obesity, vol. 33, no. 1, pp. 54-66, 2009.

[6] Q. He, Z. Gao, J. Yin, J. Zhang, Z. Yun, and J. Ye, "Regulation of HIF- $1 \alpha$ activity in adipose tissue by obesity-associated factors: adipogenesis, insulin, and hypoxia," American Journal of Physiology-Endocrinology and Metabolism, vol. 300, no. 5, pp. E877-E885, 2011.

[7] C. Jiang, A. Qu, T. Matsubara et al., "Disruption of hypoxiainducible factor 1 in adipocytes improves insulin sensitivity and decreases adiposity in high-fat diet-fed mice," Diabetes, vol. 60, no. 10, pp. 2484-2495, 2011.

[8] F. Galli, M. Piroddi, C. Annetti, C. Aisa, E. Floridi, and A. Floridi, "Oxidative stress and reactive oxygen species," Contributions to Nephrology, vol. 149, pp. 240-260, 2005.

[9] W. Wu, J. Li, M. Zhao, and X. Liu, "HIF-1 $\alpha$ mediates visfatininduced CTGF expression in vascular endothelial cells," Cellular and Molecular Biology, vol. 63, no. 4, pp. 28-32, 2017.

[10] M. M. Braun, E. A. Overbeek-Wager, and R. J. Grumbo, "Diagnosis and management of endometrial cancer," American Family Physician, vol. 93, no. 6, pp. 468-474, 2016.

[11] M. Uzunlulu, O. Telci Caklili, and A. Oguz, "Association between Metabolic Syndrome and Cancer," Annals of Nutrition and Metabolism, vol. 68, no. 3, pp. 173-179, 2016.

[12] B. Samal, Y. Sun, G. Stearns, C. Xie, S. Suggs, and I. Mcniece, "Cloning and characterization of the cDNA encoding a novel human pre-B-cell colony-enhancing factor," Molecular and Cellular Biology, vol. 14, no. 2, pp. 1431-1437, 1994.

[13] W. H.-H. Sheu, T.-M. Chang, W.-J. Lee et al., "Effect of weight loss on proinflammatory state of mononuclear cells in obese women," Obesity, vol. 16, no. 5, pp. 1033-1038, 2008.

[14] Y. Chang, D. Chang, K. Lin, S. Shin, and Y. Lee, "Visfatin in overweight/obesity, type 2 diabetes mellitus, insulin resistance, metabolic syndrome and cardiovascular diseases: a metaanalysis and systemic review," Diabetes/Metabolism Research and Reviews, vol. 27, no. 6, pp. 515-527, 2011.

[15] A. R. Moschen, A. Kaser, B. Enrich et al., "Visfatin, an adipocytokine with proinflammatory and immunomodulating properties," The Journal of Immunology, vol. 178, no. 3, pp. 1748$1758,2007$.

[16] T. Luk, Z. Malam, and J. C. Marshall, "Pre-B cell colonyenhancing factor (PBEF)/visfatin: a novel mediator of innate immunity," Journal of Leukocyte Biology, vol. 83, no. 4, pp. 804816, 2008.

[17] S. H. Jia, Y. Li, J. Parodo et al., "Pre-B cell colony-enhancing factor inhibits neutrophil apoptosis in experimental inflammation and clinical sepsis," The Journal of Clinical Investigation, vol. 113, no. 9, pp. 1318-1327, 2004. 
[18] M. H. Jarrar, A. Baranova, R. Collantes et al., "Adipokines and cytokines in non-alcoholic fatty liver disease," Alimentary Pharmacology \& Therapeutics, vol. 27, no. 5, pp. 412-421, 2008.

[19] A. Samara, M. Pfister, B. Marie, and S. Visvikis-Siest, "Visfatin, low-grade inflammation and body mass index (BMI)," Clinical Endocrinology, vol. 69, no. 4, pp. 568-574, 2008.

[20] M. Otero, R. Logo, R. Gomez et al., "Changes in plasma levels of fat-derived hormones adiponectin, leptin, resistin and visfatin in patients with rheumatoid arthritis," Annals of the Rheumatic Diseases, vol. 65, no. 9, pp. 1198-1201, 2006.

[21] T. D. Filippatos, C. S. Derdemezis, I. F. Gazi et al., "Increased plasma visfatin levels in subjects with the metabolic syndrome [3]," European Journal of Clinical Investigation, vol. 38, no. 1, pp. 71-72, 2008.

[22] H. J. Kim, Y. S. Lee, E. H. Won et al., "Expression of resistin in the prostate and its stimulatory effect on prostate cancer cell proliferation," BJU International, vol. 108, no. 2b, pp. E77-E83, 2011.

[23] S. T. Patel, T. Mistry, J. E. P. Brown et al., "A novel role for the adipokine visfatin/pre-B cell colony-enhancing factor 1 in prostate carcinogenesis," Peptides, vol. 31, no. 1, pp. 51-57, 2010.

[24] Y. Li, Y. Zhang, B. Dorweiler et al., "Extracellular nampt promotes macrophage survival via a nonenzymatic interleukin6/STAT3 signaling mechanism," The Journal of Biological Chemistry, vol. 283, no. 50, pp. 34833-34843, 2008.

[25] Y.-C. Lee, Y.-H. Yang, J.-H. Su, H.-L. Chang, M.-F. Hou, and S.-S. F. Yuan, "High visfatin expression in breast cancer tissue is associated with poor survival," Cancer Epidemiology, Biomarkers \& Prevention, vol. 20, no. 9, pp. 1892-1901, 2011.

[26] M. A. A. K. Folgueira, D. M. Carraro, H. Brentani et al., "Gene expression profile associated with response to doxorubicinbased therapy in breast cancer," Clinical Cancer Research, vol. 11, no. 20, pp. 7434-7443, 2005.

[27] Y. Wang, C. Gao, Y. Zhang et al., "Visfatin stimulates endometrial cancer cell proliferation via activation of PI3K/Akt and MAPK/ERK1/2 signalling pathways," Gynecologic Oncology, vol. 143, no. 1, pp. 168-178, 2016.

[28] T. T. Ilhan, A. Kebapcilar, S. A. Yilmaz et al., "Relations of serum visfatin and resistin levels with endometrial cancer and factors associated with its prognosis," Asian Pacific Journal of Cancer Prevention, vol. 16, no. 11, pp. 4503-4508, 2015.

[29] S. N. Avcioglu, S. O. Altinkaya, M. Küçük, H. Yüksel, I. K. Ömürlü, and S. Yanik, "Visfatin concentrations in patients with endometrial cancer," Gynecological Endocrinology, vol. 31, no. 3, pp. 202-207, 2015.

[30] W. Tian, Y. Zhu, Y. Wang et al., "Visfatin, a potential biomarker and prognostic factor for endometrial cancer," Gynecologic Oncology, vol. 129, no. 3, pp. 505-512, 2013.

[31] W. Y. Tian, Y. M. Wang, Y. F. Zhang, and F. X. Xue, "The research advances in the relationship between visfatin and cancer," Zhonghua Zhong Liu Za Zhi, vol. 39, no. 5, pp. 321-324, 2017.

[32] P. Luhn, C. M. Dallal, J. M. Weiss et al., "Circulating adipokine levels and endometrial cancer risk in the prostate, lung, colorectal, and ovarian cancer screening trial," Cancer Epidemiology, Biomarkers \& Prevention, vol. 22, no. 7, pp. 1304-1312, 2013. 


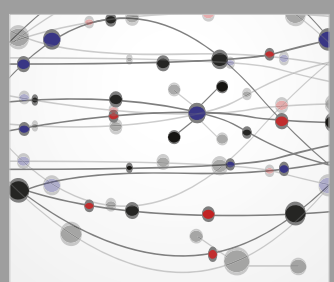

The Scientific World Journal
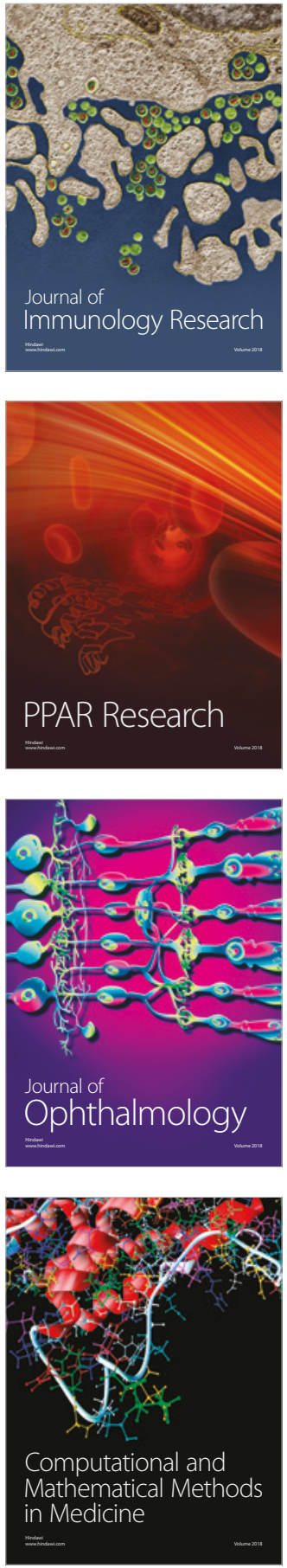

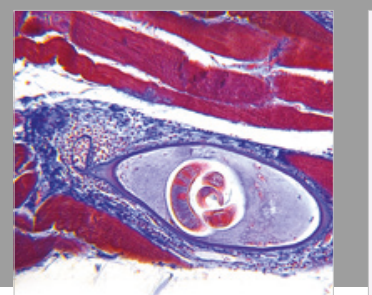

Gastroenterology Research and Practice

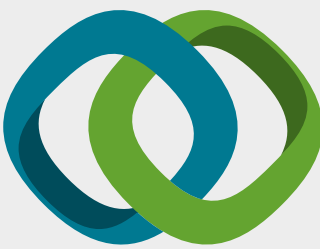

\section{Hindawi}

Submit your manuscripts at

www.hindawi.com
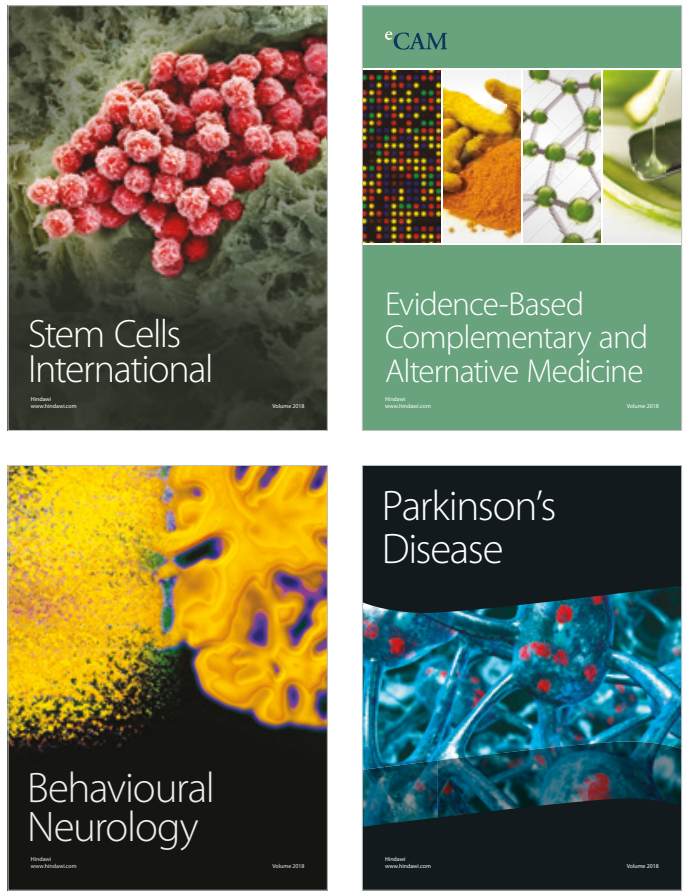

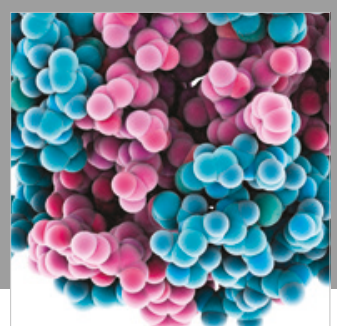

ournal of

Diabetes Research

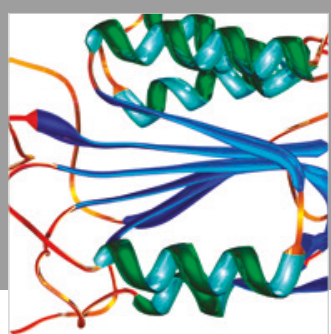

Disease Markers
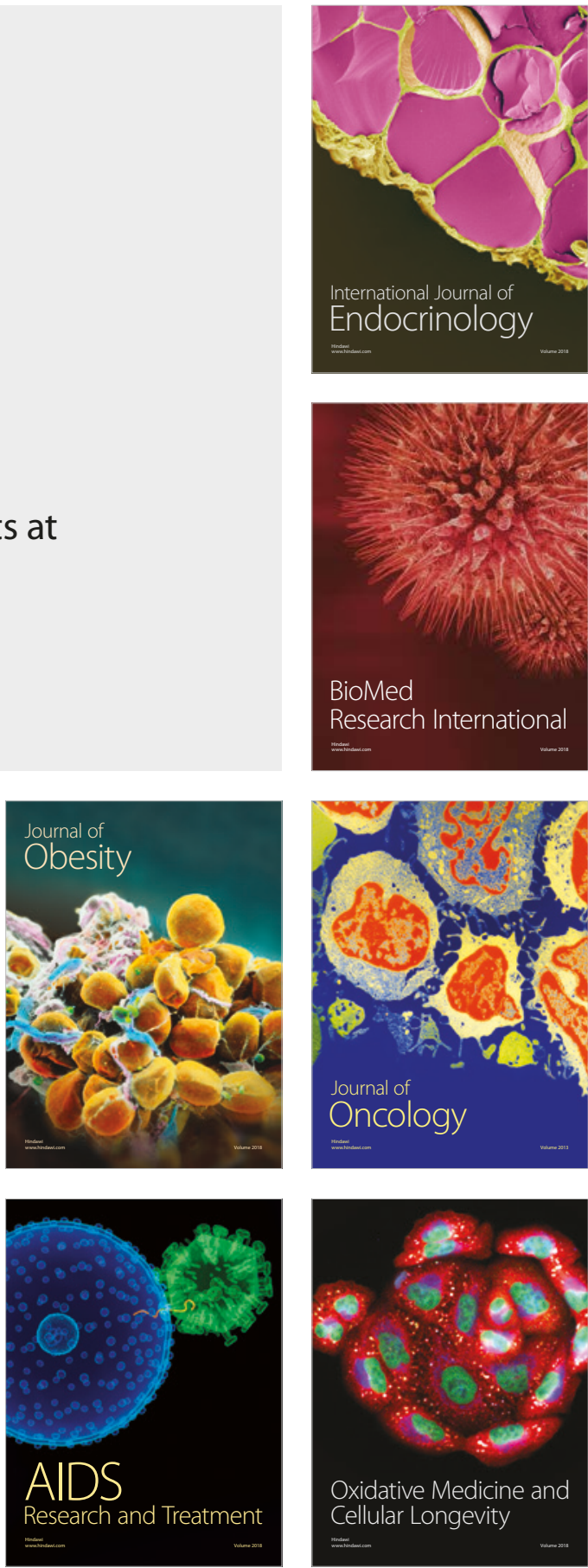